\title{
SOME PROBLEMS BIOCOMPATIBLE MATERIALS USED FOR MAKING ENDOPROSTHESES ANKLE
}

\author{
Drd. Eng. Ştefan-Cătălin Popescu1, Prof. Phd. Dr. Eng. Gheorghe I. Gheorghe², \\ Prof. Phd. Dr. Eng. Octavian Donţu ${ }^{3}$, Sl. Dr. Eng. Besnea Daniel ${ }^{4}$ \\ 1,3,4 Politehnica University of Bucharest \\ SplaiulIndependenţeinr. 313, sector 6, Bucharest \\ ${ }^{2}$ The National Institute of Research and Development \\ in Mechatronics and Measurement Technique, \\ Sos. Pantelimon, No. 6-8, Sector 2, Bucharest, Romania \\ popescu.stefancatalin@yahoo.com
}

\begin{abstract}
Biocompatible materials are used for the set ankle endoprosthesis used in modern medical practice or polymeric biomaterials, biomaterials composite and ceramic biomaterials. Ankle endoprosthesis must become an integral part of the patient's body should be accepted and tolerated by the patient, the latter passing through a process of integration and adaptation very stressful.
\end{abstract}

Keywords: Biocompatible Materials Ankle Endoprostheses.

\section{Introduction}

Biomaterials are natural or artificial materials used in biological systems.

The main types of biomaterials are:

- metals: stainless steel, cobalt alloys, titanium and titanium alloys;

- polymers: silicone rubber, acrylic resins, polyurethanes;

- ceramics: aluminium oxide, carbides, sulphides.

The choice of material for making the implant depends on many factors such as implant functionality, interaction with the patient's body type and duration of implantation.

The main problem of biomaterials is how your body accepts these materials, i.e. biocompatibility.

Artificial endoprosthetic materials come into contact with the body, which can cause reactions in the body.

Surgical implantation of these biomaterials artificial forms appropriate help restore function otherwise compromised functional ankle.

Unfortunately, these materials cannot be used long-term, such as increased wear, insufficient corrosion resistance and lack of biocompatibility. Implants are used for a longer period of time, the proper development of materials with greater longevity and biocompatibility is very important.
While many materials are currently used as biomaterials, titanium alloys are rapidly developing as the first choice for most endoprostheses.

\section{The Major Components of Ankle}

Ankle joint consists of several structures that support up to 1.5 times body weight when the person goes and up to eight times body weight when running or jumps.

The ankle is composed of two joints: the joint subtalar and ankle joint itself.

The joints are held together by muscles, ligaments, tendons and highly perfused with blood vessels and nerves.

Ankle joint consists of three bones:

- ankle called the talus bone;

- the upper surface of the talus is engaged in a recess formed in the lower ends of the tibia and fibula;

- the underside of the heel bone called the talus sits on the calcaneus.

The talus acts as a hinge into place to allow the foot to move upward (dorsiflexion) and down (plantar flexion). Figure 1 shows the ankle bone structure [1]. 


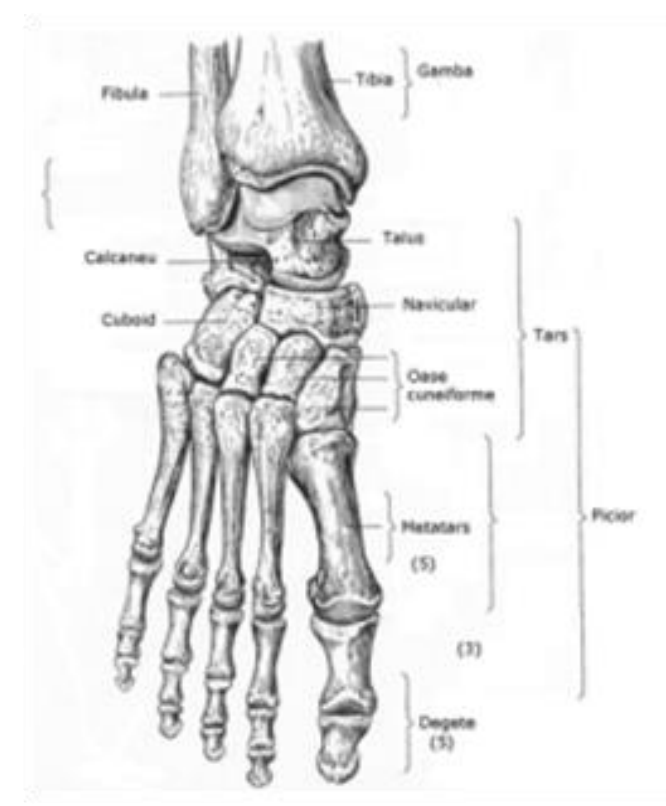

Figure 1: The ankle bone structure [1]

\section{Analysis of the Ankle Endoprosthesis Constructive}

Endoprostheses are devices or elements that are applied to a patient's body in order to be used for replacement of body parts lost for example, after an accident, illness or missing from birth to refill, correct or compensate for a deficit.

These endoprostheses are custom made from the morphological and functional characteristics of the patient, and thus can only be used by the patient.

Endoprosthesis must become an integral part of the body and be accepted by the patient, the latter passing through a real process of integration, internalization and identification, very stressful.

Achievements in the field of biomaterials based on three scientific fields: chemistry, biology and physics, and technological applications, culminating in physical clinical achievements.

Ankle endoprosthesis consists of three components. Ankle endoprosthesis components are not articulated with each other, but interact as moving parts. Ankle endo-prosthesis is coordinated by muscles and ligaments of the body. Between the joint surfaces of the plastic is mobile (PE).

In figure 2 is shown an ankle endoprosthesis model having two metal components and a polyethylene component.

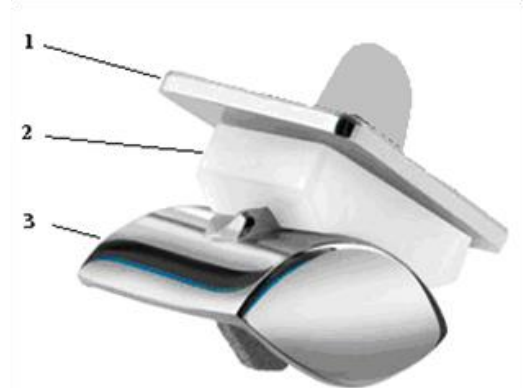

Figure 2: Ankle endoprosthesis: 1- metal component; 2- polyethylene (UHMWPE); 3- metal component;

4. The materials used for the biocompatible endoprosthesis for ankle

In order to serve for longer periods, without rejecting an implant biomaterials must have mechanical, chemical and biological comply with requirements such as biocompatibility, bioactivity and osteoinductive.

They must be inert to reduce the foreign body reaction and to reduce to a minimum the immune response.

A common classification of biomaterials is carried out based on structural metal, polymer, ceramic and composite.

The materials used in the construction of an implant must meet the following two criteria: the criterion biochemical and biomechanical criteria.

For meeting a biochemical material should be biocompatible, and the biomechanically must primarily fatigue, but this is not the only parameter. 
The first metallic material has been used in orthopedics alloys of stainless steel and cobalt chrome. fixed.

In figure 3 is shown an ankle endoprosthesis

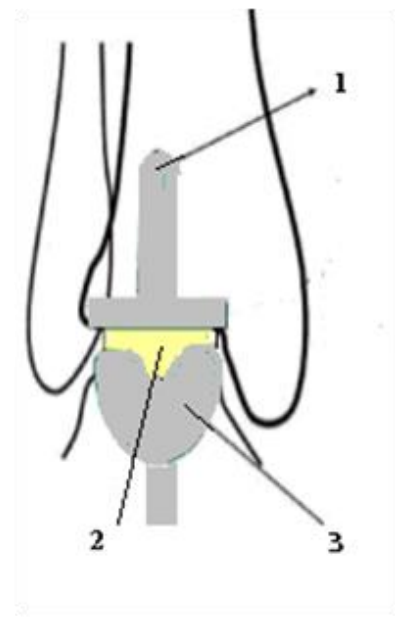

Figure 3: Endoprosthesis ankle fixed: 1-metal component; 2-polyethylene (UHMWPE); 3- metal component.

Stainless steel is resistant to a wide range of corrosive agents having a high chromium content of $12 \%$, and a protective layer of oxide, $\mathrm{Cr} 203$, resistant to corrosion.

Stainless steel is used for the manufacture of temporary used in traumatology, as well as fracture fixation plates used, and the bolts.
The first type of stainless steel used in the implants was vanadium (18-8Va), but its corrosion resistance was not so good.

In order to increase the corrosion resistance and the composition thereof was added molybdenum (188Mo), which later became 316 stainless steel.

In the 1950s, carbon component 316 stainless steel was reduced from $0.08 \%$ to $0.03 \%$ by weight, in order to increase the resistance to corrosion.

Now this is called the steel and 316L stainless steel contains an amount of $0.03 \%$ of carbon, $2 \%$ magnesium, 17-20\% chromium, 12-14\% nickel, 2$4 \%$ molybdenum, and other elements in smaller amounts, such as such as phosphorus, sulfur and silicon. The passive layer (resistant to corrosion) of these steels it is not as robust as in the case of the titanium alloys [2].

For this reason, stainless steels are used only achieve temporary medical implants such as screws and rods orthopedic fracture fixation. These steels can be work-hardened by cold working.

The stainless steel used to manufacture implants is austenitic stainless steel.

Austenitic stainless steel has a high chromium content (more than 20\%) and nickel was partially substituted by Mn with a high nitrogen content of 0.3-0.4\%, which gives improved mechanical properties and resistance to increased corrosion.

Because interaction of human tissue and biomaterial takes place at the interface between the two components, the surface properties of the implant material are of great importance Table 1 [2] [3] [4] [5].

Table 1: Composition of 316 and 316 stainless steels [2] [3] [4] [5]

\begin{tabular}{|c|c|c|c|c|}
\hline Properties & Stainlesssteel & $\begin{array}{l}\text { Co-Cr } \\
\text { alloy }\end{array}$ & Titnium & Ti-6Al-4V alloy \\
\hline Tensilestrength $\sigma_{\mathrm{T}}[\mathrm{MPa}]$ & $586-1351$ & 655-1896 & 760 & $965-1103$ \\
\hline Driplimmit $\sigma_{\mathrm{E}}[\mathrm{MPa}]$ & $221-1213$ & $448-1606$ & 485 & $896-1034$ \\
\hline Density $[\mathrm{g} / \mathrm{cm} 3]$ & 7,9 & 8,3 & 4,5 & 4,5 \\
\hline ModulusE [GPa] & 190 & $210-253$ & 110 & 116 \\
\hline Stamina $\sigma_{0}[\mathrm{MPa}]$ & $241-820$ & $207-950$ & 300 & 620 \\
\hline
\end{tabular}

The modulus of elasticity of the material presented is at least seven times higher than that of bone.

This mismatch can cause the phenomenon of "supra consolidation" a condition characterized by bone resorption in the vicinity of the implant.

Clinical complications arise from the fact that most of the mechanical stress is taken up by the implant, thus depriving the bone tissue homeostasis necessary for the mechanical stimulation.

The mechanical properties of an implant depend not only on the type of material used and the manufacturing, thermal and mechanical treatments may alter the material microstructure.
For example, in the case of cold processing (forging or Cylinder) deflections result in an increase in the hardness and strength of the material, but unfortunately reduces the ductility and increases the chemical reactivity.

The corrosion resistance of titanium and its alloys is due to the formation of an adhesive layer of titanium oxide, TiO2 entire surface. Ti6Al4V alloy is most commonly used in orthopedics.

In parallel with these conventional metallic materials, in 1960, discovered Buehler Wang NiTi alloys with shape memory effect. The effect of "shape memory" is the ability of a material to recover its shape after plastic deformation. Such shape memory 
materials are useful in the manufacture of devices for anchoring the endoprosthesis.

Although they have been used for more than 20 years in biomedical devices NiTi alloys have difficulty in use due to allergies and problems arising due to toxicity [8].

Other properties of the biomaterials studied properties relates to bioactive biomaterials which the ability to adhere to the surface of the tissue and interact to stimulate biological response.

Depending on their biocompatibility, the content is grouped into two categories: the biocompatibility intrinsic and extrinsic biocompatibility (functional) [2].

Factors determining the inherent biocompatibility are insolubility, resistance to corrosion at a pH of 6.6 to 7.5 , the electrical neutrality, mechanical strength, while the biocompatibility of extrinsic determinants are biostability, form the macroscopic structure of the surface micromorphology. In this case, the surface of the implant must be compatible with the host tissue in terms of chemical, biological and physical (including the surface morphology) [11] [12] [13].

The bioavailability of extrinsic material refers to the material's mechanical proper-ties, such as modulus of elasticity, the optimum deformation characteristics of trans-mission requests at the interface between implant and tissue. Optimum conditioning of the biomaterial and the living tissue is achieved when the surface area and structural compatibility are satisfied.

The biocompatibility of an implant depends on numerous factors such as the patient's general health, age, the permeability of the tissue, immunological factors and characteristics of the implant (the roughness and porosity of the material, chemical reactions, corrosion properties, its toxicity).

In terms of chemical, bio-compatible materials should be stable and have good resistance to corrosion, due to the peculiarities stresses to which are subject the patient:

- the composition of the internal environment varies continuously, the base having an acid character, there is also a microbiological corrosion, cannot be estimated in laboratory conditions;

- depending on the destination, the materials are subjected to constant mechanical stress, leading to corrosion under tension or cyclic, in which case the corrosion fatigue.

From the physical point of view, great importance has thermal conductivity.

Due to the lack of free electrons tissues have a low thermal conductivity, which avoids thermal shock.

Therefore, biocompatible materials are the better since their thermal conductivity is low.
After interaction with the body known bioinert materials, bioactive biotolerate, bioresorbable and hybrid.

Bioinert materials such as titanium, tantalum, alumina and polyethylene, exhibit very little chemical interaction with the surrounding tissue.

The tissues may adhere to the surface of inert material or by increasing their micro-irregularities surface (osseointegration) or by using special adhesive (acrylate).

In the long term, the latter not the ideal way of fixing the implants.

However, many polymeric implants are considered to be safe and effective for a period ranging from several months to a few years. Biological reaction is inevitable, but is compensated by the design of implants. There is an ion exchange reaction be-tween the bioactive material and body fluids which the material particles diffuse into the liquid and vice versa, while resulting in a biologically active layer of calcium phosphate, which is chemically and crystallo-graphically equivalent to the bone structure.

Bio-tolerate materials are separated from the body through an interface that is thick enough not show significant disturbance in conformance therewith.

None of the metallic materials used in orthopedics is not bioactive themselves.

In order to obtain bioactive metal implant surface may be covered with a bioactive ceramic (HA and BGs) coating; or may chemically modify the surface of the material to obtain a deposit of ceramic or bioactive in vivo to induce an interaction between the tissue and material.

Resorbable biomaterials are used in orthopaedics to replace the bone tissue, bone fracture repair, retaining ligament, meniscus and intervertebral disc.

They are marketed as rods, screws, and plates.

Among the bioabsorbable macro-molecular compounds include: PLA, PGA and PDS. Studies were performed with the material of shape memory NiTi alloys in particular, to increase the wear resistance of the porous titanium [11] [12] [13].

Co-Cr-Mo alloy is one of the most widely used implant alloys for artificial joints and provide a good combination of mechanical properties, corrosion resistance and biocompatibility. There are several types of materials currently used CoCrMo. Every material has a different microstructure, and thus, different optimized properties for a specific design or function. During the manufacture of the alloy with a high carbon content (HC), carbon precipitates to form relatively large spherical carbide inclusions in the crystalline lattice of the metal. For body composition of this alloy carbides are favored with the highest ratio of metal to carbon, such as $\mathrm{Cr}$ and Mo in the matrix to form Cr21Mo2C6. 
As a result, the immediate surrounding matrix of carbide inclusions in the chromium is poor.

This was suggested that the surface area to increase corrosion.

The microstructure of the alloy composition affects corrosion behaviour in simulated body fluids due to changes in surface chemistry.

UHMWPE is a biomaterial used for the production of the sliding element in the composition of the ankle endoprosthesis due to the reduced coefficient of friction and high resistance to mechanical stress.

The major problem is wear and the effect of wear particles on the longevity of the endoprosthesis in vivo.

Party wear particles generated from UHMWPE endoprosthesis made of osteoclasts and bone resorption triggers immediate periprosthetic tissue, culminating in loss of fixation and, finally, the revision endoprosthesis.

Due to friction between polymeric surfaces and metal surfaces, the asperity deformation of the contact area is not only elastic but also plastic.

UHMWPE is polyethylene type material which degrades over time, especially after irradiation range air.

A very important aspect in terms of material influence on biological reactions is the degradability. Polyethylene UHMWPE is highly chemically durable and therefore does not de-grade the particles of UHMWPE and persist in tissues.

The mechanical properties of UHMWPE are dependent on processing system, and the sterilization mode [9] [10].

Currently studies are to improve the wear characteristics of high-density polyethylene used in orthopaedic implants.

Using the construction of the ankle endoprosthesis crosslinked UHMWPE using crosslinking improves the wear resistance of the polyethylene.

Studies have indicated that cross-linking can reduce the wear that occurs over 95\% [10].

Cross-linked polyethylene has a better resistance of about 40 times than the conventional UHMWPE.

UHMWPE is odourless, tasteless and non-toxic.

It embodies all the features of high density polyethylene (HDPE), in addition, it is resistant to concentrated acids and bases, as well as many organic solvents.

It is highly resistant to corrosive chemicals, with the exception of oxidizing acids; it has a very low absorption of moisture and is highly resistant to abrasion.

Cracking severe manifestations of bone-implant interface, including anchoring osteolysis, aseptic and lead to the weakening of the endoprosthesis after about 15 years of implantation.

The main cause of this phenomenon is utilized in the UHMWPE particles generated from the articular surface of the polymer [10].

\section{Mechanisms of Wear}

The endoprosthesis extracted during the operations of maintenance have identified six mechanisms of wear: adhesive wear, abrasive wear, abrasive wear with the third body wear of the contact fatigue and wear of the fretting corrosion and wear.

Considering that wear is a gradual process that must be fought, were studied factors contributing to the deterioration of areas where these types of wear:

- adhesive wear;

- abrasive wear;

- wear to the third body;

- wear fatigue.

- adhesive wear, Figure 4, occurs when the connection between the two surfaces occurs so that the bond strength is stronger than the force of resistance of the material and a small part of the material is removed from the surface and is attached on the other. This process leaves small cavities at the surface and can lead to "transfer film" polymer on metal surfaces, which are occasionally observed.

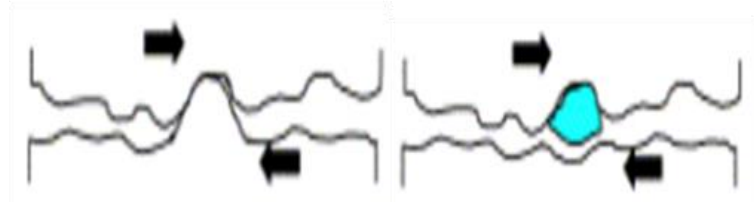

Figure 4: Lysing adhesive

- abrasive wear, Figure 5, a surface is produced as a result of mechanical action (cut-ting or scratching) of the hard particles which are free or fixed to a surface of the softer material and the other, producing wear particles. These wear particles in the form of micro stamina or fragments of material at the plastically deformed zones from one or more cycles.

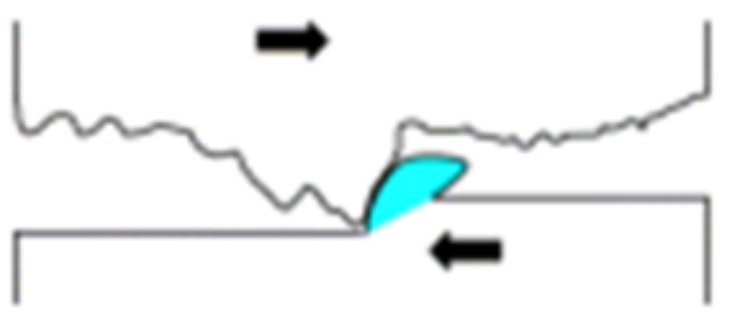

Figure 5: Abrasive wear

- wear with the third body, the destruction is caused by particles of foreign material (metal, bone) which are incorporated into the articular surfaces, Figure 6.

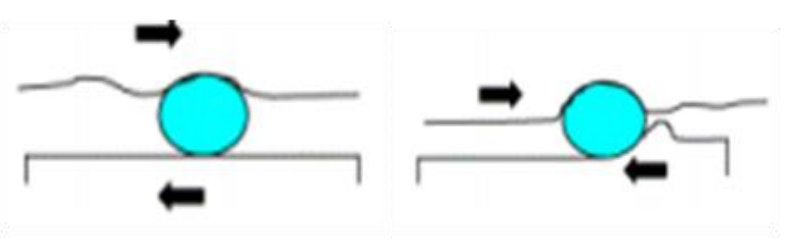

Figure 6: Lysing of the third body 
These bodies can lead to scratches and lead metal contour accelerate abrasive wear.

This type of wear has a high degree of destruction.

- wear by fatigue, Figure 7, may lead to cracks in the surface of the endoprosthesis eliminates particles that propagate on the surface. It occurs as a result of cyclical food contact surfaces, followed by plastic deformation network atomic surface layer, cracks, nicks or flaking. Generally, these appear as wear particle detachment from the material. Under the surface of the large forces they can also be caused by the third body between two articulated surfaces, which lead to accelerated wear duration.

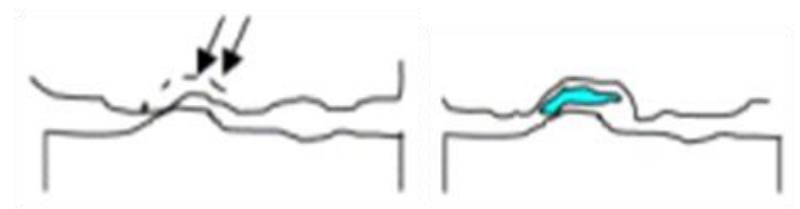

Figure 7: Wear fatigue

Fatigue cracks may grow in the polymer composition under conditions of total compressive cyclic loading, when the loading work conditions are cyclic component.

These cracks may propagate in the substrate further in accordance with the tensile, until it reaches critical size, and this can culminate in the local fracture, delamination and / or crater formation of the polymer component.

In the process of aging at an endoprosthesis these mechanisms combine, not all at once, but at different times, depending on the stresses to which is subject endoprosthesis phenomena being specific each patient.

An example would be the combination of abrasive wear - wear fatigue. This re-quires the generation of a burr by abrasion, cracking and separation followed by particles from the surface due to fatigue.

These fundamental mechanisms of wear are added one: rubbing the side surfaces, such as endoprosthesis-bone interface; friction between the surface of the polyethylene component and the metal component.

The resulting particles are listed in frictional consisting of: bone, polymethylme-thacrylate (PMMA), the metal alloy endoprosthesis, particles resulting from metal corrosion and hydroxyapatite.

In turn, these primary particles get between the friction surfaces (most of the components of the endoprosthesis) wear emphasizing at this level [10].

\section{The Importance of Choosing the Material for Making the Endoprosthesis Ankle}

Lies in the fact that the ankle joint is a hinge particularly important and demanding the role is the execution leg movements.

Due to the biomechanical behaviour of the joints, any condition arising from its lev-el but also in the foot, may have major consequences on the stability and leg mobility and therefore the entire body of the patient.

The correspondence between the articular surfaces is quite accurate. In the internal part (tibial) surfaces are almost vertical. On the external side (fibula) are curved sur-faces, oblique and down below.

Currently, the most widely used biocompatible materials used for the endoprosthe-sis for ankle as polyethylene with high molecular weight (UHMWPE) - located be-tween the two parts of metal alloys based on titanium (Ti-6Al-4V) or Co-Cr for the metal parts the endoprosthesis.

To schematically represent the consequences of a long operation ankle endoprostheses, Figure 8, as inputs and outputs represented by interactions with the external environment, such as adjacent bone tissue.

The system has functions of guiding the coupling, stopping the movement of and transmission of forces.

Transform is the support, which converted the pressure in stability (support).

Under the action of cyclic dynamic applications and complex movements per-formed in a biological environment into the endoprosthesis can lose as optimum operability.

Wear phenomena occur, which translate into deformation and wear of the endo-prosthesis components, changing the surface roughness, the occurrence of wear particles and their expulsion and synovial fluid of rejection.

This is manifested by the appearance of macrophage cells and tissue necrosis in the end, Figure 8 [14].

Movement endoprosthetic components give rise to complex application states that, ultimately, can lead to loss of stability of the endoprosthesis loosening and endoprosthesis fixation that can compromise the operability endoprosthesis and, ultimately, can lead to cracking and breaking of the bone. 


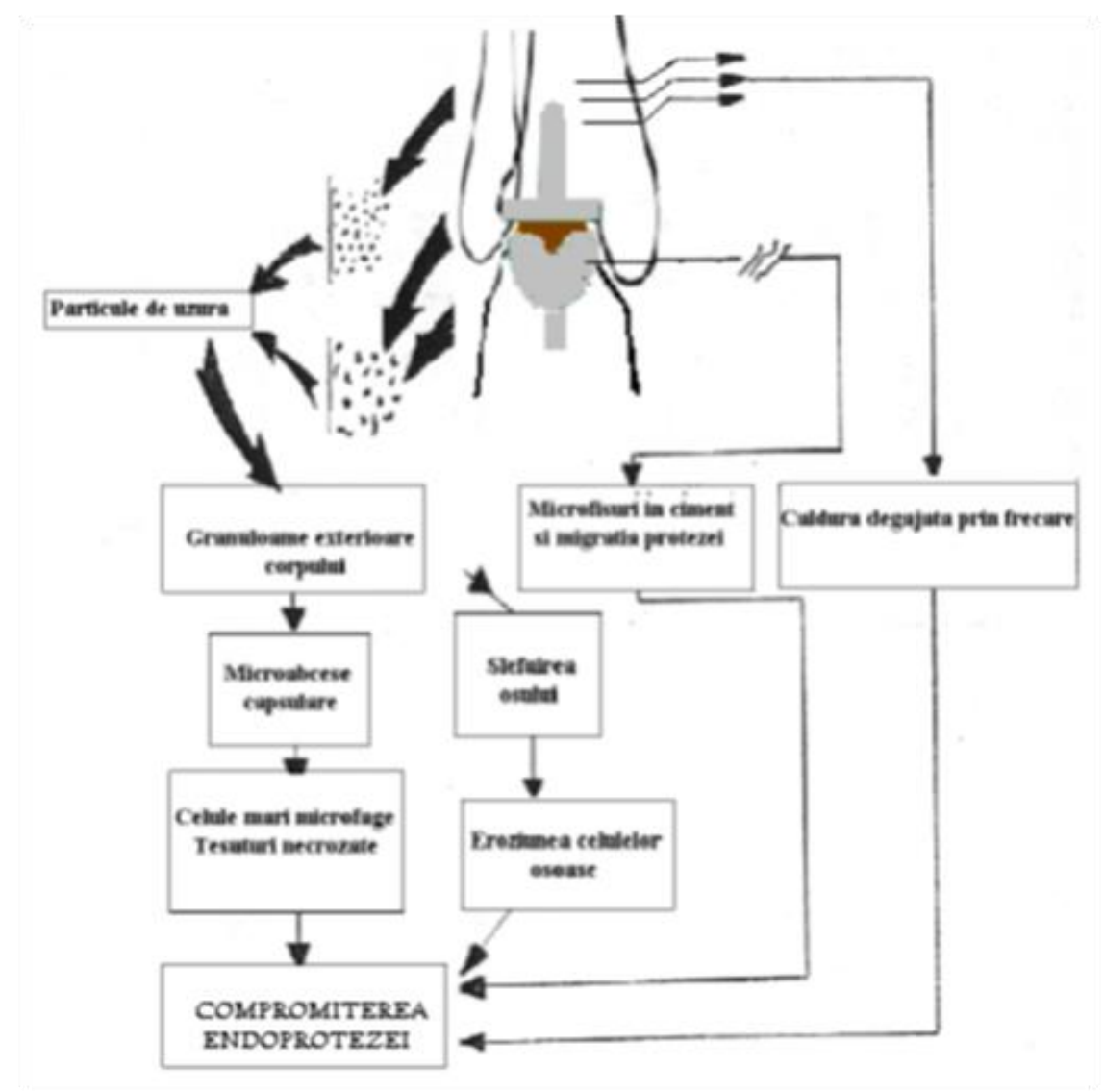

Figure 8: Schematic representation of the consequences of the operationan ankle endoprosthesis for long [14] [15]

Metallic materials such as titanium alloys have a high resistance to all forms of corrosion and good mechanical properties, have biocompatibility good, a load at break higher and an elastic limit close to that of the tensile strength, which eliminates the any permanent deformation problems in case of high stress [15].

Polymeric materials, although they have a compressive strength and good resistance to bending as high as the metal, they are biocompatible and have a low coefficient of friction and low wear, which makes them the best solution for achieving endoprosthesis ankle.

Wear a hip endoprosthesis depends on the number of cycles of friction which is subject to and not how much time sitting in the patient. Attrition rate of endoprosthesis varies greatly from one patient to another because each patient is very different activity.

An individual activity average is about 1 million yards / year, and most active reach 3.2 million cycles / year.

Persons older, less active, are 0.2 to $0,500,000$ cycles / year [14] [15].

Wear ankle endoprosthesis are the result of complex interactions between embodiment, patient characteristics and medical factors related to the surgical act of implantation.

\section{Conclusions}

Ankle endoprosthesis intended to replace body parts or remedy deficiencies of their dysfunction if properly designed and made of suitable biomaterials have been well surgical instrument positioned and fixed within the time of their use has increased significantly.

The task of adjusting biomaterials for integration purposes and regeneration of tissue after implantation is a challenge for the future. It is however necessary to introduce a biological evaluation of these modified surfaces as well as in vitro assays using cell cultures.

\section{References}

[1] Alexandru,P., Diaconescu, D.,Ştefan, I.: Structural modeling mechanisms articulated ankle human foot (Modelări structural prin mecanisme articulate a gleznei piciorului uman), ANNALS of the ORADEA UNIVERSITY, Fascicle of Management and Technological Engineering, Volume VI (XVI), 2007: 1070.

[2] Branemark, P.I, Breine, U, Johansson, B., Roylance, P.J, Röckert, H., Yoffey,J.M.: Rege- 
neration of bone marrow, Acta Anat. 59:146(1964).

[3] Buehler, W.J, Wang, F.E: A summary of recent research on the Nitinol alloys and their potential application in ocean engineering, Ocean Eng. 1:105-120(1967).

[4] Charnley, J.: Anchorage of the femoral head prosthesis to the shaft of the femur. J. Bone Joint Surg. 42B:28-30(1960).

[5] Chu, Y, Dai, K., Zhu, M., Mi, X.: Medical applications of NiTi shape memory alloy in China, Mater. Sci. Forum. 327-328:55-62 (2000).

[6] Ciccone, W., Motz, C., Bentley, C., Tasto,J.: Bioabsorbable implants in orthopaedics: new developments and clinical applications, J. Am. Acad. Orthop. Surg. 9:280-288(2001).

[7] Navarro, J.M., Michiardi, A., Castaño, O. and Planell, J.A.: Biomaterials in orthopaedics, J R Soc Interface. Oct 6, 5(27): 1137-1158 (2008).

[8] Ratner, B.D., Hoffman, A.S., Schoen, F.J.: Biomaterials science. An introduction to materials in medicine, 2nd edn. Elsevier/Academic Press; Amsterdam, The Netherlands/New York, NY: (2004).

[9] Swanson, A.B.: Silicone rubber implants for replacement of arthritic or destroyed joints in the hand, Surg. Clin. North Am. 48:1113-1127(1968).
[10] Ducheyne, P., Radin, S., Heughbaert, C.: Calcium phosphate ceramic coating on porous ti-tanium: effect of structure and composition on electrophoretic deposition, vacuum sintering and in vitro dissolution. Biomaterials, 11:244254(1990).

[11] Evans, S.L., Gregson, P. J.: Composite technology in load-bearing orthopaedic implants, Biomaterials, 19: 1329-1342 (1998).

[12] Hench, L., Polak, J.: Third generation biomedical materials, Science. 295:1014-1017(2002).

[13] Long, M., Rack, H.J.: Titanium alloys in total joint replacement-a materials science perspective, Biomaterials. 19:1621-1639(1998).

[14] Navarro, JM., Michiardi, A., Castaño, O. and Planell, J.A.: Biomaterials in orthopaedics, J R Soc Interface. Oct 6, 5(27): 1137-1158 (2008).

[15] Gheorghe,I.Gh., Badita, L.L.: Experiments and surfaces characterization of the femoral heads of hip prostheses, Digest Journal of Nanomaterials and Biostructures, Vol. 7, No 1, , 279 - 285Ianuarie - Martie(2012).

[16] Capitanu, L., Badita, L.L. and Bursuc, D. C.: Damage of the Co-CrMo Femoral Head of a Total Hip Prosthesis and Its Influence on the Wear Mechanism, Journal of Materials Science and Engineering A1 662-670(2011). 